ISSN: 1794-9998 / Vol. 3 / No. 1 / 2007 / pp. 109-119.............

\title{
Violencia política: algunas consideraciones desde la psicología social ${ }^{*}$
}

\section{Political violence: some considerations from social psychology}

\author{
Idaly Barreto* \\ Universidad Católica de Colombia \\ Fundación Universitaria Konrad Lorenz \\ Bogotá, Colombia \\ Henry Borja \\ Corporación Universitaria Iberoamericana \\ Bogotá, Colombia \\ Recibido: diciembre 15 de 2006 \\ Revisado: enero 18 de 2006 \\ Aceptado: enero 26 de 2007
}

\section{Resumen}

Este trabajo presenta algunas cuestiones importantes desde la psicología social para la explicación de la violencia política; entre ellas, se destaca la importancia de los grupos humanos como actores principales del conflicto intergrupal y las creencias grupales como elemento central de análisis en la legitimación de la violencia política. El planteamiento pretende dilucidar la necesidad que tienen los grupos armados de realizar construcciones ideológicas con el propósito de justificar sus propias acciones.

Palabras clave: legitimación, violencia política, conflicto intergrupal, creencias.

\section{Abstract}

This work presents some important questions from Social Psychology for the explanation of the political violence. Among them one stands out the group importance of the human groups like film stars of the intergroup conflict and beliefs like central element of analysis in the legitimation of the political violence. The exposition tries to explain the necessity that has the armed groups to make ideological constructions in order to justify their own actions.

Key words: legitimation, political violence, intergroup conflict, beliefs.

El presente trabajo hace parte de la línea de investigación en Psicología Política del Grupo de Investigación de Psicología Social y Política de la Universidad Católica de Colombia.

* Correspondencia: Idaly Barreto, Doctorado en Psicología Social, docente Facultad de Psicología, Universidad Católica de Colombia y Fundación Universitaria Konrad Lorenz. Dirección postal: Facultad de Psicología, Universidad Católica de Colombia, Calle 47 № 13-32. Bogotá, Colombia. Correo electrónico: mibarreto@ucatolica.edu.co. Henry Borja, Doctorado en Psicología Social, docente Facultad de Psicología, Corporación Universitaria Iberoamericana. Correo electrónico: henryborjaorozco@gmail.com. 


\section{Introducción}

Observando la realidad social y política que se vive actualmente en el mundo, se puede apreciar que, como en el pasado, la violencia política continúa siendo un medio para dominar a otros y establecer, cambiar o preservar determinado orden social. Esta constante relación entre poder y violencia hace que la violencia política sea un tema complejo con consecuencias sociales, políticas y psicológicas, entre otras, de vital importancia para los seres humanos. Visto de esta manera, la necesidad y posibilidad de estudiar este fenómeno desde diferentes perspectivas teóricas resulta interesante para los investigadores de las ciencias sociales y humanas.

Por esto, y teniendo en consideración que la violencia política ha sido una constante de la historia colombiana, es esencial asumir desde la psicología social un compromiso con la explicación y construcción de una sociedad en la que el ordenamiento social y político se pueda mantener y reformar a través de canales de participación democrática; es decir, por vías pacíficas y dialogadas. Por tal razón, desde esta perspectiva, se hará especial énfasis en el papel que desempeñan los grupos humanos como protagonistas de esa constante lucha por el mantenimiento, reforma o cambio de determinado orden social.

De esta manera, siguiendo la tesis planteada por Sabucedo sobre la legitimación de la violencia política, cuyo planteamiento central es que cuando un grupo social decide emplear la violencia como estrategia de acción política es necesario que ésta se legitime, dado que el impacto psicológico que generan las acciones violentas implica consecuencias contrarias a las esperadas por el grupo (Sabucedo, Rodríguez \& Fernández, 2002). Es decir que en lugar de cumplir con los objetivos políticos a través del uso de la violencia, el grupo se enfrenta al rechazo social que genera el uso de esta estrategia.

En este sentido, además del carácter instrumental, la aprobación o rechazo social a la violencia política depende principalmente de la legitimación que se haga de ella. Esta justificación ideoló- gica es la estrategia principal de los grupos para intentar obtener en algunos segmentos de la sociedad la aceptación del grupo y la aprobación de sus acciones políticas violentas.

En este orden de ideas, el objetivo del presente artículo es brindar una explicación psicosocial de la violencia política teniendo presente la estrecha relación entre la psicología de la legitimidad, el conflicto intergrupal y las implicaciones del discurso como medio de difusión para los procesos de legitimación y deslegitimación.

\section{Sobre el concepto de legitimación}

Aunque el concepto de legitimidad puede ser relativamente nuevo para la psicología empírica, éste ha jugado un papel importante en disciplinas como la filosofía social y política (Jost y Major, 2001). Respecto al ámbito de la psicología, en la década de los noventa se desarrolló el auge en esta línea de investigación, principalmente en 1993 y 1994 con la publicación de un amplio número de artículos y libros en psicología social, cuyo tema era la legitimidad aplicada al estudio de la ideología, la justicia y las relaciones intergrupo; todos motivados principalmente por las teorías de identidad social, dominancia social y sistema justificacional o sistema de legitimación.

Enmarcados en la disciplina de la psicología, los conceptos de legitimidad e ilegitimidad han sido empleados para explicar fenómenos sociales como: estatus, desigualdad, justicia, desviación y control social; movimientos sociales, especialmente protesta social; cambio social y desarrollo y difusión de nuevas normas, actitudes, prácticas; y forma institucional.

Entre los temas de investigación destacados se encuentran:

- Los procesos cognitivos y perceptuales involucrados en la valoración de la legitimidad (Crandall y Beasley, 2001; Yzerbyt y Rogier, 
2001; Robinson y Kray, 2001).

- La tolerancia a la injusticia entre miembros de grupos desventajados (Olson y Hafer, 2001; Major y Schmader, 2001; Ellemers, 2001; Wright, 2001).

- La legitimación de la desigualdad tomando como base los conceptos de estereotipos e ideología, integrados con las teorías de identificación social, dominancia social y sistema de justificación (Ridgeway, 2001; Glick y Fiske, 2001; Sidanius, Levin, Federico y Pratto, 2001; Spears, Jetten y Doosje, 2001; Jost, Burges y Mosso (2001).

- Los procesos institucionales y organizacionales de la legitimación (Elsbach, 2001; Tyler, 2001; Jackman, 2001).

Todas estas líneas de investigación coinciden en que la cuestión clave es la necesidad de las personas por construir racionalizaciones ideológicas que provean justificación para sus propias acciones y la de los demás. En consecuencia, se han desarrollado teorías de la psicología de la legitimación (procesos intraindividuales de legitimación), sus efectos en otros procesos psicológicos (como identificación, comparación y atribución) y sus efectos en procesos sociales (violación de derechos humanos y normas establecidas) (Zelditch, 2001; Kelman, 2001).

En este orden de ideas, los estudios sobre psicología de la legitimación juegan un papel fundamental para la psicología social y política, pues las actitudes, creencias y estereotipos sirven para legitimar el orden social y proveen el soporte ideológico para el sistema social y político. En este sentido, es reiterativa la relevancia conceptual de la legitimidad para explicar el origen y mantenimiento de la violencia política en una sociedad, y responder a cuestiones claves como ¿en qué forma los violentos justifican sus conductas y demuestran que ellos actúan de manera legítima? o ¿por qué algunas personas justifican conductas violentas que traspasan las normas morales y sociales?
Por otra parte, aunque el concepto de legitimación se aplica extremadamente bien en psicología social, éste no ha sido tratado sistemática-mente (Jost y Major, 2001), probablemente porque teorizar un concepto involucrado en diferentes procesos sociales y en muchos niveles de organización social es una tarea compleja. Esta variedad de fenómenos explicados por la legitimación sugiere que es un tipo de proceso auxiliar que explica la estabilidad de alguna clase de estructura, en algún nivel, que emerge y es mantenido por algún otro proceso social básico (las cursivas son del autor, Zeltditch, 2001). Desde esta perspectiva, la definición de legitimación y deslegitimación como proceso puede proporcionar una definición general que agrupe todos estos contextos.

En el capítulo "Reflection on social and psychological processes of legitimization and delegitimization", Kelman (2001, p. 57) define legitimación como

El proceso de recategorizar una acción, política o demanda -o un sistema, grupo o persona- que fue previamente ilegítima y ahora se convierte en legítima". Y deslegitimación como "el proceso inverso de recategorización, mientras que fue previamente legítimo ahora se convierte en ilegítimo.

Esta definición es una importante contribución para el análisis de la conducta social (poder, autoridad, influencia social, acción colectiva, cambio político), porque permite analizar útilmente la legitimación y deslegitimación como procesos de recategorización y explicar cambios en las normas sociales que facilitan a subgrupos de una sociedad justificar violaciones extremas de las normas sociales, tales como el asesinato político o el uso de la tortura.

Asumiendo esta definición como punto de referencia conceptual, y siguiendo las consideraciones de Bar-Tal (1990), Apter (1997), Kelman (2001) y Zeltditch (2001), se plantean cinco aspectos relevantes para la aplicabilidad del concepto de legitimación al estudio de la violencia política (Barreto, 2004): 
1. Los procesos de legitimación y deslegitimación son claves para explicar el mantenimiento de la violencia política en algunos grupos de la sociedad. De acuerdo con lo anterior, cuando un grupo utiliza la violencia se debe enfrentar como mínimo a dos clases de consecuencias: positivas y negativas. En relación a la primera, al margen del carácter instrumental de la violencia política (consecución de objetivos de parte de los grupos que la ejercen), ésta se mantiene principalmente por la capacidad que tienen los grupos para legitimarla. En segundo lugar, los grupos se ven enfrentados al rechazo social inherente al impacto negativo que conlleva una acción violenta. En este caso, es de esperar que el uso de la violencia disminuya. Sin embargo, en algunas ocasiones los grupos optan por la estrategia de deslegitimación del adversario con el propósito de reducir el impacto emocional en algunos sectores de la sociedad y así mantener legítimamente el uso de la violencia.

2. Los procesos de legitimación y deslegitimación son conjuntamente útiles cuando el propósito es explicar que cuando una persona o grupo es deslegitimado, la violencia contra esta persona o grupo es legitimada. En un conflicto social, normalmente los valores morales y sociales inhiben las acciones violentas, por lo que es de esperar que los grupos que emplean la violencia como medio de acción política la legitimen necesariamente recurriendo a una estrategia discursiva que contenga la deslegitimación del adversario. Kelman y Hamilton (1989) han identificado tres procesos sociales que facilitan la participación de las personas en acciones violentas: cuando las acciones son explícitamente ordenadas y aprobadas por las autoridades legítimas (autorización) cuando las acciones se transforman en rutina como parte de acciones programadas tanto a nivel individual como organizacional (rutinización) y cuando excluyen al objetivo de estas acciones como miembro o actor moral de la comunidad (deshumanización).

3. Estudiar como elementos centrales las creencias en el proceso de recategorización de un grupo o una acción. Las creencias se relacionan con la ideología que un grupo necesita para construir racionalizaciones que provean justificación para sus propias acciones y la de los demás. Sabucedo, Rodríguez \& Fernández (2002) han trabajado sobre las creencias legitimadoras de la violencia política, entre las que se encuentran la referencia a la existencia de un conflicto, la situación en la que se encuentra el grupo, la presentación del grupo como defensor de las vías dialogadas y pacíficas, y el victimismo del endogrupo como elementos básicos para justificar las acciones violentas, pues en la medida en que se logre ese respaldo social, se irá legitimando esa forma de actuación del grupo.

Como se puede apreciar, las creencias mencionadas anteriormente evidencian, tal y como lo plantea Van Dijk (2003), que el lenguaje del grupo se caracteriza habitualmente por una estrategia que favorece a los miembros del grupo y a la presentación positiva de éste. Básicamente las categorías que definen de forma positiva el discurso político de la agrupación están relacionadas con los aspectos sociales del grupo, esto es, los aspectos históricos, sociales, políticos y culturales que comparten los miembros del grupo y definen la ideología.

Por el contrario, pero con el mismo objetivo de preservar su imagen positiva, cuando el grupo se ve obligado a asumir la responsabilidad de sus acciones, opta por referirse a ellas en términos jurídicos o militares propios de un ejército estatal (De la Corte, Moreno y Sabucedo, 2004). Así, encontramos que los grupos armados se refieren a los secuestros como detenciones, a los asesinatos como ejecuciones o bajas en las filas del adversario; éstos son sólo unos pocos ejemplos de lo que se puede encontrar en el lenguaje militar 
que emplean los grupos armados.

Esta estrategia de deslegitimar al adversario ha sido estudiada en el contexto colombiano por el Grupo de Investigación en Psicología Política de la Universidad de Santiago de Compostela (Barreto, 2004; Borja, 2004; Sabucedo \& otros, 2004; Sabucedo, Barreto, Borja, De la Corte \& Durán, 2006). Dichos estudios plantean e investigan la manera como las creencias son atribuidas a otro grupo con el propósito de incluirlo en categorías sociales extremadamente negativas para ser excluidos de los grupos humanos que actúan dentro de los límites de normas y/o valores aceptables para la sociedad. En esencia, la deslegitimación niega la humanidad del grupo categorizado, para ello recurre a categorías como deshumanización, proscripción, caracterización de rasgos, uso de rótulos políticos, comparación de grupos (BarTal, 1996b, 2000; Worchel, Cooper, Goethals \& Olson, 2002).

4. Es importante destacar la propuesta de Kelman (2001) dirigida a estudiar los procesos de legitimación y deslegitimación como fenómeno social, causados e impulsados por fuerzas operantes en todas partes de la sociedad y propagados a través de diferentes medios de comunicación e influencia. En consecuencia, es de esperar que la violencia como estrategia de acción política, para cambiar o mantener la estructura política de una sociedad, necesariamente cuente con una ideología que legitime las acciones violentas del grupo, que las proscriba como políticas y deslegitime al adversario. De tal manera que los grupos violentos se ven en la necesidad de recurrir al discurso como medio de influencia social para legitimar acciones violentas; de lo contrario, algunos sectores sociales no las aprobarían.

5. Considerando la cuestión anterior, se debe destacar que de los intereses y preferencias de las personas o grupos - contexto culturaldepende un cambio rápido y congruente en las normas de la sociedad. Esto es, asumiendo que el discurso es una estrategia que acompaña la acción política, es de esperar que los grupos que ejercen la violencia han de tratar por todos los medios a su alcance de difundirlo ante la mayor parte de la sociedad. No obstante, esto no es garantía de que el total de la población apruebe dichos actos, si se tiene en cuenta que la sociedad está expuesta a múltiples fuentes de información que transmiten discursos provenientes de diferentes sectores sociales que pueden influir en la aceptación o rechazo de las acciones de grupos armados. Aun en el caso de que la audiencia tenga acceso limitado a otros discursos, y la credibilidad y persuasión de los violentos puede ser tal que muchos subgrupos sociales adopten sus creencias, tampoco pueden asegurar que las personas justifiquen sus acciones como ocurre con algunas comunidades indígenas y otros grupos sociales en Colombia.

De los cinco aspectos mencionados anteriormente, los tres primeros tienen que ver directamente con los procesos psicológicos involucrados en los procesos de legitimación y deslegitimación; los dos últimos están relacionados con la difusión de creencias a través del discurso.

\section{Conflicto intergrupal}

Asumiendo que las creencias son un elemento clave en la legitimación, y definiendo violencia como "una acción, o estado o situación, que se genera siempre y se cualifica de manera exclusiva en el seno de un conflicto" (Aróstegui, 1994, p. 29), es importante analizar este fenómeno desde una perspectiva psicosocial con el propósito de identificar los procesos intergrupales que le permiten a un grupo armado asumir y promover creencias a algunos segmentos de la sociedad, presentando así una realidad social y psicológica del conflicto que le facilite legitimar el uso de la violencia en un contexto político. Por ello, referirse a los grupos humanos como protagonistas del conflicto es una cuestión necesaria. 
Según Bar-Tal (1990, p. 41), grupo "es una colectividad de individuos con un sentido definido de pertenencia y creencias compartidas, incluyendo las creencias de grupo; las cuales regulan su conducta al menos en materia relacionada con lo colectivo". Este concepto contempla lo que Bar-Tal considera tres condiciones necesarias y suficientes para que una colectividad sea un grupo, éstas son: 1 . los individuos de una colectividad se definen a sí mismos como miembros de un grupo; 2. ellos comparten creencias, incluyendo las creencias de grupo; y 3. tienen algún nivel de actividad coordinada. Estas tres características grupales se consideran suficientes para iniciar la argumentación sobre el papel de los grupos sociales en el conflicto político y, por lo tanto, en la violencia política.

La definición de grupo planteada por Bar-Tal resalta la importancia de la pertenencia a un grupo por parte de un individuo, lo cual, sin lugar a duda, es determinante para la conducta intergrupal. De acuerdo con Tajfel (1984, p. 275), "toda la conducta mutua de dos o más individuos está determinada por su pertenencia a diferentes grupos o categorías sociales". Esta cuestión es particularmente importante para una explicación psicosocial sobre el porqué de la violencia política ejercida por grupos, si se tiene presente que los miembros de un grupo cometen acciones violentas en función de sus respectivas pertenencias a un grupo.

Un ejemplo de ello son las acciones violentas que le son asignadas como objetivo a los miembros de un grupo armado en contra del adversario, o cualquier enfrentamiento armado entre la guerrilla contra soldados del ejército o grupos paramilita-res. En estos casos, es de esperar que las acciones violentas de los miembros de un grupo contra miembros de otro(s) grupo(s) estén determinadas por el hecho de pertenecer a la categoría de adversario.

Cuando el adversario es el Estado, la categoría puede ser tan amplia (soldados del ejército, representantes políticos, funcionarios, etc.) que las acciones violentas se pueden dar contra personas que están inmersas en el conflicto y, en muchos casos, ni siquiera están relacionadas directamente con las demandas políticas del grupo armado. El caso de algunas familias campesinas es un ejemplo de esta situación. Ellas son obligadas a tomar partido por los diferentes actores del conflicto y al ser consideradas colaboradoras de alguno de los grupos son incluidas en la categoría de adversario. Esta polarización del conflicto supone, además, que un gran número de personas en situación indefensa se vea expuesto a las retaliaciones propias de un grupo armado.

El ejemplo anterior evidencia que para ejercer la violencia política es fundamental que los individuos asuman la creencia "somos un grupo". Para ello es necesario, según la definición psicosocial de pertenencia a un grupo propuesta por Tajfel (1984, p. 265), que los miembros de un grupo sean considerados como tales cuando se autocategoricen con un alto grado de aprobación dentro de un determinado modo de comportarse, y cuando los otros estén de acuerdo en categorizarlos dentro de ese mismo comportamiento. Definida así, la pertenencia a un grupo es necesaria para la existencia del mismo. Si los miembros del grupo no comparten esta creencia, entonces no se consideran miembros del grupo y, por lo tanto, no podrán desplegar una actividad coordinada (Morales, 1999, p. 284).

Pero aunque la pertenencia es una característica esencial para el surgimiento de un grupo, así como para las actividades que se puedan desarrollar en él, la legitimación del uso de la violencia, además, se fundamenta en ciertas creencias que el grupo está preparado para adquirir (creencias de grupo). Éstas se constituyen en la segunda característica y son definidas por Bar-Tal (1990, p. 36) como convicciones (a) que los miembros de un grupo son conscientes de compartir y (b) a las que consideran definitorias de su 'pertenencia grupal'". Las creencias de grupo, según este autor, contemplan dos elementos importantes a la hora de definirlas.

El primer elemento de esta definición sugiere que las creencias han de ser compartidas por los miembros del grupo. Según Bar-Tal (1990), los miembros del grupo comparten, por lo menos, dos creencias. Una hace referencia a cualquier contenido que es 
objeto de la creencia grupal, y la otra se refiere al conocimiento concreto que afirma que la anterior es compartida por los miembros del grupo. En el segundo elemento, las creencias grupales se consideran como definidoras de la esencia del grupo y juegan un papel decisivo para la identidad social. De hecho, este mismo autor afirma que "las creencias grupales sirven de base para la formación del grupo y posteriormente como ligazón para la existencia del grupo" (Bar-Tal, 1996a, p. 257).

A partir de la definición de creencias de grupo propuesta por Bar-Tal, que se fundamenta en la pertenencia y creencias de un grupo, se puede decir que los grupos que ejercen la violencia necesitan de las creencias de grupo. En primer lugar de la creencia fundamental "somos un grupo"; en segundo lugar, de creencias adicionales que sean funcionales para el grupo. Y aunque las creencias adicionales -metas, valores, historia, normas- pueden variar de un grupo a otro, éstas proporcionan identidad a los miembros del grupo. Además, ciertas creencias adicionales compartidas acerca del grupo (y del exogrupo) cumplen con la función de legitimar las acciones violentas que en él se desarrollan.

En coherencia con lo anterior, y de acuerdo con autores como Bar-Tal (1990), Bar-Tal, Kruglanski \& Klar (1989), Pruitt \& Rubin (1986), cuando las creencias varían de un grupo a otro y éstas, a su vez, son percibidas como una amenaza por los miembros de un grupo; la contradicción entre las creencias de dos o más grupos puede conducir al conflicto y la confrontación. Esta amenaza puede ser percibida cuando la incompatibilidad con otro(s) grupo(s) conlleva a que la consecución de metas u objetivos propios del grupo sea bloqueada o impedida por otro grupo. Así pues, el conflicto intergrupal comienza con la percepción de que las metas, intenciones y/ o acciones propias del grupo son incompatibles con las de otro grupo.

Lo anterior es común y frecuente de encontrar en espacios donde se desarrollan los conflictos políticos; además, es fundamental para la explicación de la violencia política, si se tiene en cuenta que las creencias sirven para legitimar el ordenamiento social y proveen el soporte ideológico para el sistema social y político (Jost y Major, 2001). Según Tilly (1998, p. 30), el conflicto político incluye en todas las ocasiones que:

- Un grupo de personas realice reivindicaciones colectivas públicas visibles sobre otros actores (reivindicaciones que si se cumpliesen afectarían los intereses de estos últimos).

- Al menos una de las partes afectadas por las reivindicaciones, incluyendo terceras partes, es un gobierno.

En este orden de ideas, cuando uno a más grupos percibe las condiciones sociales reguladas por el Estado como ilegítimas, pero susceptibles de cambio,

la percepción de ilegitimidad determinará probablemente, más pronto o más tarde, intentos de cambiar la situación, y la percepción de inestabilidad (que puede interpretarse como el desarrollo dentro de un grupo de la conciencia de alternativas cognitivas a la situación existente) es probable que se asocie, más pronto o más tarde, con una disminución de la percepción dentro del grupo de la legitimidad de la situación (Tajfel, 1984, p. 281).

Como resultado de esta situación, es de esperar que la combinación de creencias grupales sobre la ilegitimidad de ciertas condiciones sociales, en conjunto con la creencia de que con la acción colectiva el grupo puede contribuir a crear o impedir el cambio social, impulse al grupo social a desarrollar la tercera característica para que una colectividad sea considerada un grupo: tener algún nivel de actividad coordinada.

Es de esperar, por lo tanto, que los grupos que ejercen la violencia creen un discurso social que contenga creencias grupales que enmarquen la situación social como injusta con el propósito de convertir la percepción de injusticia en motivo para participar y legitimar la violencia política; además, 
es obvio que incluyan creencias grupales que responsabilicen al grupo adversario de la situación en la que se encuentra y, por ende, lo deslegitime. Este último aspecto, es considerado por Rodríguez, Fernández y Sabucedo (1999, p. 44) necesario para la politización de la identidad, pues "la atribución de responsabilidades al exogrupo que ostenta el poder o la autoridad (ellos) adquiere una clara dimensión político-reivindicativa y, por lo tanto, posiblemente movilizadora".

Así pues, el conflicto político que desencadena en la confrontación violenta se acompaña necesariamente por la acción estratégica del grupo dirigida a construir un discurso que promueva creencias que preparen y mantengan a los miembros del grupo, y algunos sectores de la sociedad, en disposición de cometer y legitimar acciones extremas, como asesinatos indiscriminados, detenciones en masa o inclusive genocidio con el propósito, según el endogrupo, de disminuir la amenaza y el peligro que el adversario representa.

\section{Discusión}

Para concluir este artículo, es importante reflexionar sobre la importancia psicosocial que tiene estudiar el fenómeno de la violencia política, dada la creciente necesidad de comprender y explicar un tema que por el impacto que genera en diferentes áreas -psicológica, social, política, etc.interesa cada vez más a las ciencias sociales. El planteamiento realizado sobre la relevancia de las creencias en el conflicto intergrupal y en la legitimación de la violencia política pone de manifiesto la necesidad de formular y desarrollar propuestas de investigación dirigidas a analizar el discurso, pues no sólo se pueden identificar las estrategias retóricas de los grupos que recurren a la violencia como estrategia de solución a los conflictos socio-políticos, sino que además permite la posibilidad de prever el uso de la violencia y reflexionar sobre el papel que desempeñan los diferentes grupos en la construcción de la sociedad.
Pues así como los grupos armados crean marcos que hacen viable la justificación de la violencia como instrumento para la obtención de objetivos políticos, de la misma manera los esfuerzos estratégicos de los grupos sociales pueden estar dirigidos a crear marcos de convivencia y tolerancia social que hagan posible el rechazo social a la violencia como estrategia de cambio social. Pero no sólo el rechazo o la ausencia de la violencia política va constituir la paz en Colombia, se necesita, además, que existan condiciones reales de igualdad social y protección de los derechos de los ciudadanos que garanticen la convivencia pacífica de la sociedad colombiana.

\section{Referencias}

Apter, D. (1997). The legitimization of violence. New York: University Press.

Aróstegui, J. (1994). Violencia, sociedad y política: la definición de la violencia. Ayer, 13, 17-55.

Barreto, I. (2004). Análisis textual del discurso legitimador de las Farc-Ep. Tesis de Grado de Doctorado. Santiago de Compostela: Universidad de Santiago de Compostela.

Bar-Tal, D. (2000). Shared Belief in a Society. Social Psychological Analysis. Estados Unidos: Sage publications..

Bar-Tal, D. (1996a). Las creencias grupales como expresión de la identidad social. En: Morales, J., Páez, D., Deschamps, J. \& Worchel, S. identidad social: aproximaciones psicosociales a los grupos y a las relaciones entre grupos (pp. 255-287). España: Promolibro.

Bar-Tal, D. (1996b). Societal beliefs in times of intractable conflict: The Israeli case. Revista de Psicología Social Aplicada, 6, 3, 65-102. 
Bar-Tal, D. (1990). Groups Beliefs. A Conception for Analysing Group Structure, Processes, and Behaviour. New York: Springer Verlang.

Bar-Tal, D., Kruglanski, A. W. \& Klar, Y. (1989). Conflict termination: An epistemological analysis of international cases. Political Psychology, 10, 233-255.

Borja, H. (2004). Legitimación de la violencia política: paramilitarismo y Estado. Tesis de Grado de Doctorado. Santiago de Compostela: Universidad de Santiago de Compostela.

Crandall, C. \& Beasley, R. (2001). A Perceptual Theory of legitimacy: Politics, Prejudice, Social Institutions, and Moral Value. En: Jost, J. \& Major, B. (Eds), The psychology of legitimacy: Emerging Perspectives on Ideology, Justice, and Intergroup Relations, pp. 77-102. United Kingdom: Cambridge University Press.

De la Corte, L., Moreno, F., \& Sabucedo, J. M. (2004). Dimensiones psicosociales del terrorismo. En: De la Corte, L., Blanco, A., Sabucedo, J. M. (Eds), Psicología y derechos humanos, pp. 189-220. Barcelona: Icaria.

Ellemers, N. (2001). Individual Upward Mobility and the Perceived Legitimacy of Intergroup Relations. En: Jost, J. \& Major, B. (Eds), The psychology of legitimacy: Emerging Perspectives on Ideology, Justice, and Intergroup Relations, pp. 205-222. United Kingdom: Cambridge University Press.

Elsbach, K. (2001). The Architecture of Legitimacy: Constructing Accounts of Organizational Controversies. En: Jost, J. \& Major, B. (Eds), The psychology of legitimacy: Emerging Perspectives on Ideology, Justice, and Intergroup Relations, pp. 391-415. United Kingdom: Cambridge University Press.

Glick, P. \& Fiske, S. (2001). Ambivalent Stereotypes as Legitimizing Ideologies: Differentiating
Paternalistic and Envious Prejudice. En: Jost, J. \& Major, B. (Eds), The psychology of legitimacy: Emerging Perspectives on Ideology, Justice, and Intergroup Relations, pp. 278-306. United Kingdom: Cambridge University Press.

Jackman, M. (2001). License to Kill: Violence and Legitimacy in Expropriative Social Relations. En: Jost, J. \& Major, B. (Eds), The psychology of legitimacy: Emerging Perspectives on Ideology, Justice, and Intergroup Relations, pp. 437-468. United Kingdom: Cambridge University Press.

Jost, J., Burges, D. \& Mosso, C. (2001). Conflicts of Legitimation among Self, Group, and System: The Integrative Potential of System Justification Theory. En: Jost, J. \& Major, B. (Eds), The psychology of legitimacy: Emerging Perspectives on Ideology, Justice, and Intergroup Relations, pp. 363-390. United Kingdom: Cambridge University Press.

Jost, J. \& Major, B. (Eds.). (2001). Introduction. Emerging Perspectives on the Psychology of Legitimacy. En: Jost, J. \& Major, B. (Eds.), The psychology of legitimacy: Emerging Perspectives on Ideology, Justice, and Intergroup Relations, pp. 3-32. United Kingdom: Cambridge University Press.

Kelman, H. (2001). Reflections on Social and Psychological Processes of Legitimization and Delegitimization. En: Jost, J. \& Major, B. (Eds), The psychology of legitimacy: Emerging Perspectives on Ideology, Justice, and Intergroup Relations, pp. 54-76. United Kingdom: Cambridge University Press.

Kelman, H. \& Hamilton, V. (1989). Crimes of obedience: Toward a social psychology of authority and responsibility. Yale: University Press.

Major, B. \& Schmader, T. (2001). Legitimacy and the Construal of social Disadvantage. En: 
Jost, J. \& Major, B. (Eds), The psychology of legitimacy: Emerging Perspectives on Ideology, Justice, and Intergroup Relations, pp. 176-204. United Kingdom: Cambridge: University Press.

Morales, J. (1999). Grupos. En: Morales, J. (Coord). Moya, M., Pérez, J., Fernández, I., Fernández, J. M., Huici, C., Páez, D. \& Marques, J, Psicología social (2 ${ }^{\mathrm{a}}$ ed.) España: Mc. Graw-Hill.

Olson, J. \& Hafer, C. (2001). Tolerance of Personal Deprivation. En: Jost, J. \& Major, B. (Eds), The psychology of legitimacy: Emerging Perspectives on Ideology, Justice, and Intergroup Relations, pp. 157-175. United Kingdom: Cambridge University Press.

Pruittt, D. G. \& Rubin, J. Z. (1986). Social Conflict. New York: Random House.

Ridgeway, C. (2001). The emergence of Status Beliefs: From structural Inequality to Legitimizing Ideology. En: Jost, J. \& Major, B. (Eds), The psychology of legitimacy: Emerging Perspectives on Ideology, Justice, and Intergroup Relations, pp. 257-277. United Kingdom: Cambridge University Press.

Robinson, R. \& Kray, L. (2001). Status versus Quo: Naive Realism and the Search for Social Change and Perceived Legitimacy. En: Jost, J. \& Major, B. (Eds), The psychology of legitimacy: Emerging Perspectives on Ideology, Justice, and Intergroup Relations, pp. 135-156. United Kingdom: Cambridge University Press.

Rodríguez, M., Fernández, C. \& Sabucedo. J. M. (1999). Identidad colectiva y movimientos sociales. Revista de Psicología Social Aplicada. 9, 3, 39-52.

Sabucedo, J. M., Barreto, I., Borja., De la Corte, L. \& Durán. (2006). Legitimación de la violencia y contexto: análisis textual del discurso de las Farc-Ep. Estudios de Psicología. 27, 3, 279-
291.

Sabucedo, J. M., Barreto, I., Borja, H., López, W., Blanco, A., De la Corte, L. \& Durán, M. (2004). Deslegitimación del adversario y violencia política: el caso de las FARC y las AUC en Colombia. Acta Colombiana de Psicología. 12, 69-85.

Sabucedo, J. M., Rodríguez, M. \& Fernández, C. (2002). Construcción del discurso legitimador del terrorismo. Psicothema. 14, 72-77.

Sidanius, J., Levin, S., Federico, C. \& Pratto, F. (2001). Legitimizing Ideologies: the Social Dominance Approach. En: Jost, J. \& Major, B. (Eds), The psychology of legitimacy: Emerging Perspectives on Ideology, Justice, and Intergroup Relations, pp. 307-331. United Kingdom: Cambridge University Press.

Spears, R., Jetten, J. \& Doosje, B. (2001). The (Il)legitimacy of Ingroup Bias: From Social Reality to Social Resistance. En: Jost, J. \& Major, B. (Eds), The psychology of legitimacy: Emerging Perspectives on Ideology, Justice, and Intergroup Relations, pp. 332-262. United Kingdom: Cambridge University Press.

Tajfel, H. (1984). Grupos humanos y categorías sociales. Barcelona: Herder.

Tilly, C. (1998). Conflicto político y cambio social. En: Ibarra, P. \& y Tejerina, B. (Eds). Los movimientos sociales: transformaciones políticas y cambio cultural, pp. 26-38. Madrid: Trotta.

Tyler, T. (2001). A Psychological Perspective on the Legitimacy of Institutions and Authorities. En: Jost, J. \& Major, B. (Eds), The psychology of legitimacy: Emerging Perspectives on Ideology, Justice, and Intergroup Relations, pp. 416-436. United Kingdom: Cambridge University Press.

Van Dijk, T. A. (2003). Ideología y discurso. Barcelona: Ariel Lingüística.

Worchel, S., Cooper, J., Goethals, G. \& Olson, J. 
(2002). Psicología Social. México: Thomson.

Wright, S. (2001). Restricted Intergroup Boundaries: Tokenism, Ambiguity, and the Tolerance of Injustice. En: Jost, J. \& Major, B. (Eds), The psychology of legitimacy: Emerging Perspectives on Ideology, Justice, and Intergroup Relations, pp. 257-277. United Kingdom: Cambridge University Press.

Yzerbyt, V. \& Rogier, A. (2001). Blame It on the Group: Entitativity, Subjective Essentialism, and Social Attribution. En: Jost, J. \& Major, B. (Eds), The psychology of legitimacy: Emerging Perspectives on Ideology, Justice, and Intergroup Relations, pp. 103-134. United
Kingdom: Cambridge University Press.

Zeltditch, M. (2001). Theories of legitimacy. En: Jost, J. \& Major, B. (Eds), The psychology of legitimacy: Emerging Perspectives on Ideology, Justice, and Intergroup Relations, pp. 33-53. United Kingdom: Cambridge University Press. 